\title{
Análisis perceptivo auditivo de la voz de las docentes de la Facultad de Ciencias de la Educación - UNIFÉ
}

\author{
Auditory perceptual analysis of the voice of the teachers of the Faculty of \\ Education Sciences - UNIFÉ
}

\author{
María Teresa Granda Tiravanti ${ }^{1}$ \\ Fara Clara Maldonado Sánchez ${ }^{2}$
}

\section{RESUMEN}

El objetivo del trabajo fue determinar cómo se encuentra la calidad vocal de las docentes de la Facultad de Educación de la UNIFÉ.

La presente investigación es un estudio descriptivo simple. La muestra consta de 35 docentes universitarios cuyas edades fluctúan entre 20 y 73 años; y entre 04 y 53 años de servicio.

Se evaluó con una encuesta y un protocolo perceptivo auditivo y de forma individual.

El análisis perceptivo auditivo evaluó: a) Pitch, b) Loudness, c) Tiempo máximo de fonación, d) Calidad vocal de las participantes.

En la mayoría de las docentes no se encontraron trastornos vocales. Se evidencia que algunas docentes presentaron Pitch grave, así también docentes con loudness fuerte y débil. En cuanto al tiempo máximo de fonación, presentaron un promedio por debajo de lo estandarizado. En cuanto a la calidad vocal la mayoría de los docentes presentaron una voz adecuada, sin embargo, se encontraron docentes con voz ronca, susurrada e infantilizada. En la relación del tiempo de servicio y la calidad vocal no se encontró relación con estos parámetros. La mayoría de docentes corresponden al $77.1 \%$ obtuvieron parámetros vocales dentro de los límites normales.

Algunas docentes cuentan con alteraciones vocales que no tendrían relación al tiempo de servicio profesional.

\section{Palabras clave}

Docentes, calidad vocal, pitch, loudness, tiempo máximo de fonación.

\section{ABSTRACT}

The objective of the present work was to determine how the voice quality of the teachers of the Faculty of Education of UNIFÉ is found.

This present research is a simple descriptive study. The sample has 35 university teachers whose ages oscillate among 20 and 73 years; and between 04 and 53 years of service.

Licenciada en Educación Primaria en la Universidad San Martín de Porras, con especialidad en audición y lenguaje, complejo microfacila, tartamudez y disfluencias y voz en la Universidad Católica del Perú (Cepal). Ha publicado diversos artículos y ponencias. Docente universitaria desde hace 27 años en la UNIFÉ. Desde hace 9 años Coordinadora del Centro de evaluación, diagnóstico y tratamiento de Lenguaje y Apredizaje.

2 Licenciada en Ciencias de la Educación "Enrique Guzmán y Valle". Especialista en audición, lenguaje, motricidad orofacial y problemas de aprendizaje.

Docente de la Universidad Peruana Cayetano Heredia. Coordinadora de Prácticas Profesionales - internado del Hospital Naval: Universidad Peruana Cayetano Heredia, Federico Villarreal y Universidad Femenina del Sagrado Corazón. 
A survey and an auditory perceptual protocol were evaluated individually.

The auditory perceptual analysis evaluated: a) Pitch, b) Loudness, c) Maximum phonation time, d) Vocal quality of the participants.

In most of the teachers we found vocal disorders. It is evident that some teachers introduced severe Pitch, as well as teachers with strong and weak loudness. With regard to the maximum phonation time, they presented an average under the standard. In terms of vocal quality, most teachers presented a right voice; nevertheless, teachers were found with hoarse, whispered and infantilized voice. In the relation of the service time and the voice quality, there was no relation with these parameters. Most of the teachers correspond to $77.1 \%$ obtained vocal parameters within normal limits.

\section{Keywords}

Teachers, voice quality, pitch, loudness, maximum phonation time.

\section{Introducción}

La voz puede definirse como un sonido voluntario producido por los pliegues vocales, gracias a la acción del soplo espiratorio ampliado y modulado por las cavidades de resonancia y regulado por el sistema auditivo. Como las aulas universitarias son grandes y las alumnas adultas, además, la dificultad en relación a los ruidos ambientales (ruidos externos e internos) demandan mayor esfuerzo vocal en las docentes universitarias (Behlau, Suzigan y Nagaro, 2004).

Nuestro deseo es que sirva este estudio como un aporte, para realizar actividades de prevención en casos de alteración por el uso y/o abuso vocal inherente a su profesión, ya que en la práctica profesional continua muchas docentes presentan dificultades de voz que interfiere así su labor cotidiana.

\section{Origen y evaluación del término de voz}

El exceso del uso de la voz hablada es característica en la actividad docente. En 1966 la Organización del Trabajo y la Organización Mundial de la Salud vienen reconociendo en los países la vigencia de la salud a los docentes, sin embargo, sigue existiendo dificultades para el reconocimiento de enfermedades vocales profesionales. En el siglo XX, las alteraciones de la voz de los docentes, pasaron a considerarse dentro de los trastornos ocupacionales al vincularse el grado de disfonía con el tipo de trabajo realizado. La vOz es un instrumento de trabajo por excelencia del maestro de escuela y muchas de las inasistencias docentes son producto de problemas de voz.

En los países desarrollados la diversificación en actividades educativas donde se imparte clases, se consideran como factores causales de riesgo, para la seguridad y salud en el sector educativo que antes no se contemplaba (Frías 2012). Asimismo, se han realizado diversos trabajos de investigación sobre la voz del docente en el Perú y el extranjero.

La voz es producida con la participación de diversas estructuras que compone el llamado tracto vocal, que comienza en la laringe y termina en la cavidad de la boca y nariz. El sonido producido por la laringe es modelado en el tracto vocal y las cavidades de resonancia. Estas cavidades proyectan la voz.

\section{Las cualidades de la voz}

- Pitch o frecuencia. Ferreira y Silva (2002) es la sensación psicoacústica relacionada al tono de voz en una persona. Puede varia de grave a aguda. 
- Laudness o Intensidad. Ferreira y Silva (2002) Es la sensación psicoacústica de la intensidad, que nos permite juzgar un tono considerado como débil o fuerte, también como potencia de voz.

- Tiempo máximo de fonación. Behlau y Pontes (1995). Es una medida de 25 a 35 segundos para hablantes y 15 a 25 segundos para hablantes femeninos.

\section{Clasificación de voces}

- Voz adaptada

Según Behlau et al. (2004), la voz adaptada es resultado de un acto fonatorio equilibrado, proviene de un sonido producido por la laringe a través de la vibración de los pliegues vocales, con el pasaje de la corriente de aire continua y eficiente.

- Voz profesional

De acuerdo a Dragone (2207), la voz profesional es modificada según las necesidades y funciones específicas de las diversas profesiones y categorías.

- Voz ronca

Behlau (2001) manifiesta que la voz ronca es un indicador alteraciones vocales. La calidad de la voz de tipo ruidoso, indica irregularidad de vibración de los pliegues vocales, presenta soplosidad y aspereza, estando alterada o disminuida la frecuencia y la intensidad.

- Voz soplada

Es la voz acompañada de aire no sonorizada por los pliegues vocales teniendo la presencia de ruido en la fonación. Es de intensidad baja y frecuencia grave.

- Voz infantilizada

Presenta un tono agudo que no corresponde a la edad del hablante. Puede aparecer en ambos sexos. Generalmente está asociada a la evaluación de la laringe y anteriorización de la lengua.

\section{Higiene vocal del docente}

Perazzo (1956) y María Behlau, María Luisa Suzigan y Lucía Nagaro (2004) coninciden en cómo evitar el mal uso de la intensidad, tono y la calidad vocal de la voz en diferentes circunstancias y lugares.

Factores ambientales. Son elementos importantes en la salud vocal, independientemente al profesor sus clases, sus técnicas, su personalidad.

Tamaño del aula, disposición del mobiliario, de las carpetas, pizarra, escritorio del profesor y el espacio para circular deben ser estudiados para la acústica del aula.

Cigarrillo. Al fumar se aspira nicotina que pasa por los pliegues vocales y pulmones donde se almacena. Con el tiempo pueden surgir lesiones malignas o benignas. Por causa del cigarrillo, la voz es más gruesa (grave).

Bebidas alcohólicas. Influyen negativamente en el volumen y la articulación de la palabra. Genera esfuerzo muscular.

Alimentación. Los granos, frutas y verduras hacen bien a la salud en general. En cambio, los alimentos de difícil digestión pueden producir reflujo gastroesofágico.

Hidratación. Tomar agua lubrica los pliegues vocales. Para el docente es aconsejable el tener una jarra con agua a temperatura ambiente y beber pequeños sorbos de agua durante el dictado de clase.

Postura corporal. Hay ciertas posturas que son usadas y pueden generar tensión o desequilibrio postural. En caso del docente, sugerimos especial atención a la altura de la cadera con relación a la mesa o escritorio, forma como se inclina para corregir los trabajos de los alumnos. También considerar 
la altura de la pizarra y el modo de borrarla manteniendo el cuello equilibrado para evitar la tensión muscular y la tensión vocal.

Reposo vocal. Usar momentos de descanso en los intervalos entre las clases buscando descansar la voz.

Carraspera. Evitar el acto de limpiado de garganta, puede irritar los pliegues vocales.

Gritar. Al gritar los pliegues vocales por la tensión exagerada y la fricción de los pliegues vocales se edematizan.

Mal uso y abuso vocal. Son hábitos conocidos como fonotrauma, que afectan la calidad de la voz.

Competencia sonora. Es hablar compitiendo con ruidos del ambiente o queriendo sobrepasar el habla de los interlocutores.

Hablar fuera de tono. Muchas personas modifican su tono de voz para causar efecto emocional en el oyente, porque les importa conseguir determinada respuesta con su voz. El hablar fuerte o débil puede perjudicar el aparato fonador, por el uso inadecuado.

\section{Alteraciones de voz más frecuentes de los docentes}

1. Fonastenia

Es el cansancio vocal de la voz (Dr. Emilio Vásquez Ayllón, 1998)

\section{Nódulos}

Según Frías (2007) es el engrosamiento en la unión del tercio anterior con los dos tercios superiores posteriores, en la zona central de la porción vibrátil de las cuerdas vocales de mayor impacto. Predomina en las mujeres.

3. Quiste

De acuerdo a Behlau et al (2004) Pequeña lesión circular unilateral.

4. Cáncer de laringe

Frías (2007) Es el segundo en incidencias del tracto aerodigestivo superior. El $80 \mathrm{y}$
90\% de los cánceres de cabeza y cuello se atribuye al consumo de tabaco y alcohol, a esto se puede agregar otros como la falta de higiene dental, tabaco, trabajo en madera, exposición a níquel, radiaciones y virus de Epstein Barra, laringitis crónica o las papilomitis.

\section{Método}

La presente investigación es un estudio descriptivo simple cuyo objeto fue determinar cómo se encuentra la calidad vocal de las docentes de la Facultad de Ciencias de la Educación de la UNIFÉ.

\section{Población y Muestra}

La muestra estuvo conformada por 35 sujetos femeninos docentes de Educación de la Universidad Femenina del Sagrado Corazón, de la Escuela de Educación Especial en sus diversos niveles cuyas edades fluctúan entre 20 y 73 años y 04 a 53 años de servicio.

\section{Material e Instrumento}

Se utilizó un protocolo de evaluación a partir de los datos de evaluación, en el cual se consideraron a evaluar los parámetros pitch, loudness, calidad vocal y tiempo máximo de fonación. Se les explicó a las docentes seleccionadas, en qué consistía la prueba, el material que se iba a emplear y cómo se aplicaría.

La evaluación se realizó en forma individual y ambiente cerrado.

Cada una de las especialistas evaluadoras escuchó las voces de las docentes y llenaron unas fichas de vaciado con la apreciación correspondiente.

Para el análisis de los resultados se establecieron frecuencias y porcentajes de las variables evaluadas, asimismo se utilizó el programa estadístico SPSS, para establecer el nivel de relación y el nivel de significancia entre las variables examinadas, edad, pitch, loudness, calidad vocal y tiempo máximo de fonación. 


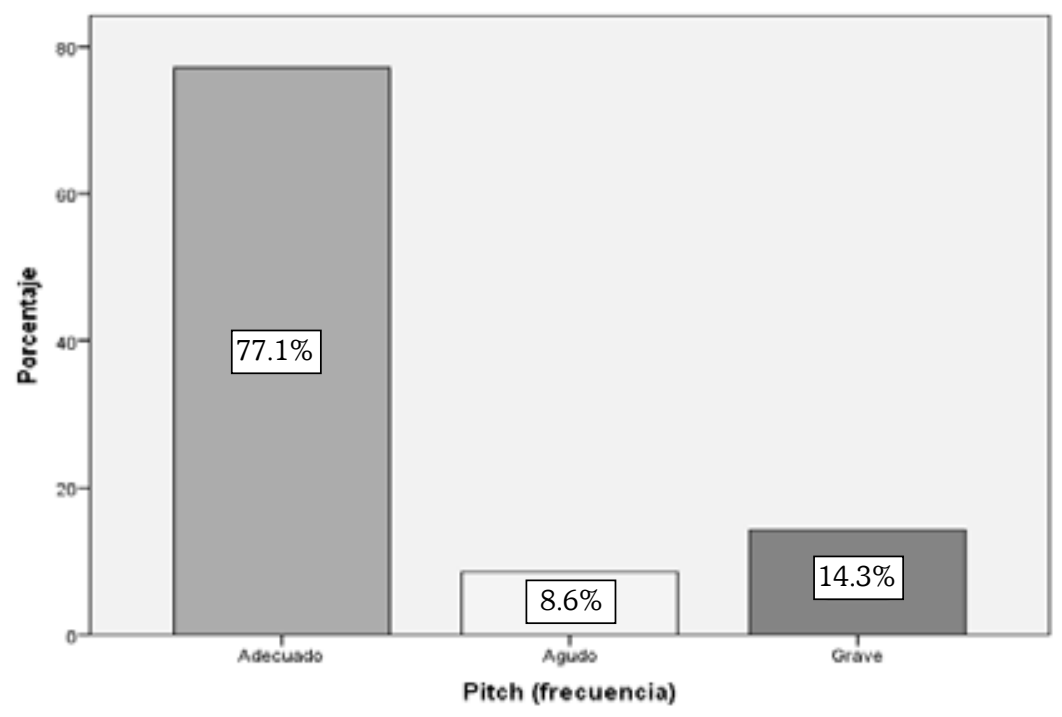

Figura 1. Nivel de pitch - Frecuencia

Diversos autores han manifestado que el pitch (frecuencia) es la característica individual y varía de acuerdo a la anatomía de la laringe, edad y sexo del individuo.

Asimismo, el $14.3 \%$ de los sujetos tuvieron un nivel de pitch grave. Para Lesile Piccoloto Ferreira y Marta A. de Andrade e Silva (2002) las voces graves son las más aceptables. El 8.6\% de los sujetos presentaron tono agudo, según las autoras mencionadas el pitch agudo transmite alegría, dinamismo y llama la atención del oyente.

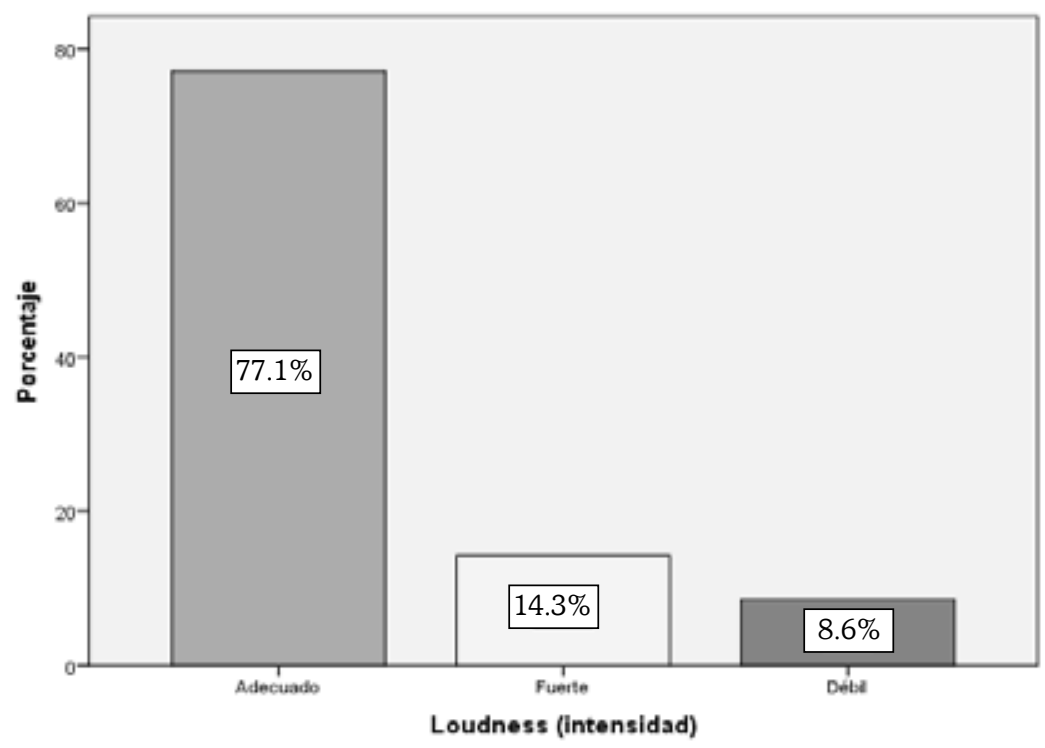

Figura 2. Nivel de loudness - Intensidad

El $77.1 \%$ de las docentes evaluadas tuvieron un nivel de laudness (intensidad) adecuado, lo que implica un apropiado funcionamiento para una presión de la columna de aire asociada, amplitud y vibración de las cuerdas vocales más específicamente la resistencia de la glotis que ofrece el pasaje de aire.
Igualmente, el 14.3 de las profesoras evaluadas tuvieron el nivel laudness y un tono fuerte, de otro lado, también se presentó el $8.6 \%$ con una intensidad débil. Según Leslie Piccolotto Ferreira y Marta Andrade e Silva (2002), el laudness es la sensación psicoacústica de la intensidad que nos permite juzgar un tono como débil o fuerte. 


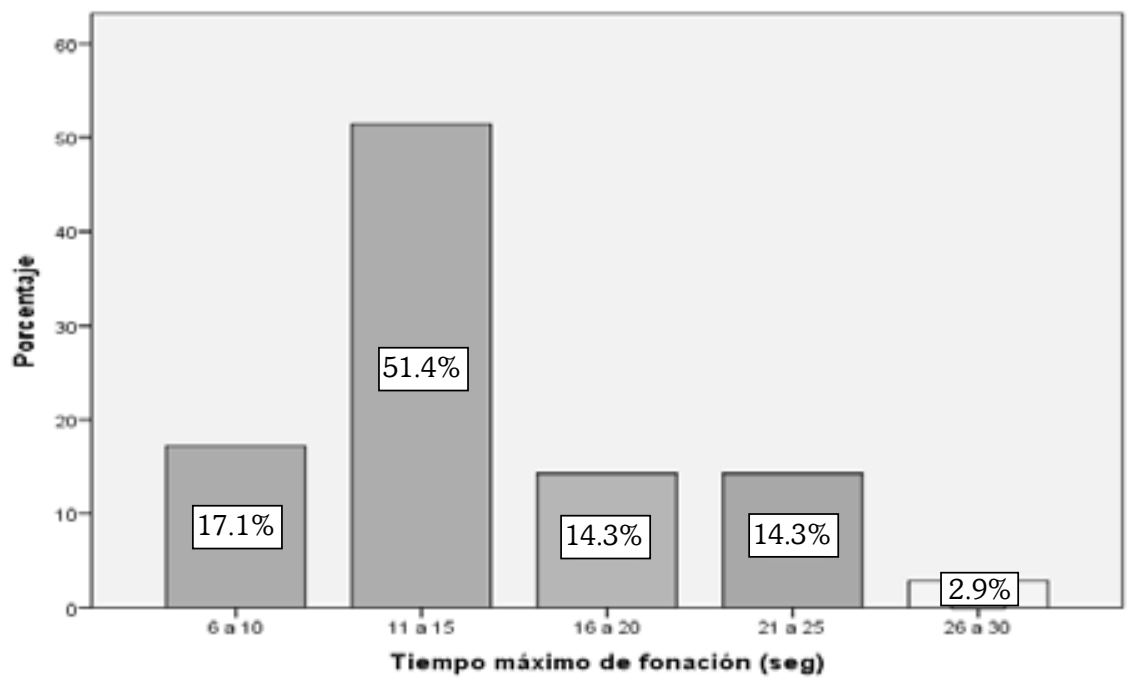

Figura 3. Nivel de tiempo máximo de fonación (TMF)

Más de la mitad de las profesoras evaluadas tuvieron entre 11 y 15 segundos de tiempo máximo de fonación (TMF), equivalente al $51 \%$ del total de sujetos. El $17.1 \%$ de las profesoras tuvieron entre 6 y 10 segundos. El $14.3 \%$ de los sujetos tuvieron entre 16 a 20 segundos y el mismo porcentaje de profesoras tuvieron entre 21 a 25 segundos como TMF.
Solo una profesora tuvo entre el 26 a 30 segundos $(2.9 \%)$.

Mara Behlau y Paola Pontes (1995) consideran como tiempo máximo de fonación de 15 a 25 segundos en la mujer. Este parámetro en los sujetos evaluados no se cumplió.

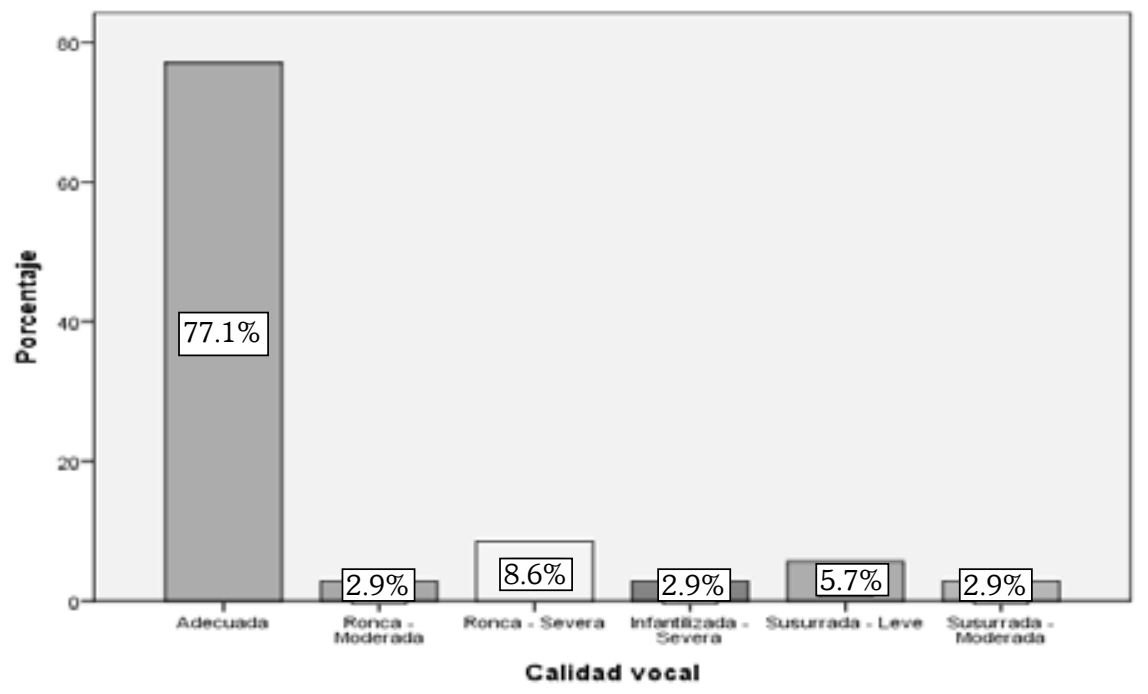

Figura 4. Nivel de calidad viocal

La mayoría de las docentes evaluadas tuvieron una calidad vocal adecuada con $77.1 \%$. Según Mara Behlau y Paolo Pontes (1995) la cualidad Vocal es el término actualmente utilizado para designar el conjunto de características que identifican una voz humana. El $8.6 \%$ de las docentes tienen voz ronca (severa) y voz ronca (moderada) en un 2.9\%. Para Mara Behlau (2001) es una cualidad de tipo ruidoso. Indica irregularidad de pliegues vocales. Se encuentra alterada la frecuencia y la intensidad.

Una docente presentó voz infantilizada (severa) 2.2\%. Según Mara Behlau (2001) presenta tono agudo que no corresponde a la edad del hablante o a la madurez psicoemocional. Otras docentes presentaron voz susurrada (moderada) 2.90\%. Mara Behlau y Paolo 
Pontes (1995) manifiestan que esta voz transmite la impresión de que quiere contar secretos, confiere un carácter de intimidación a la emisión.

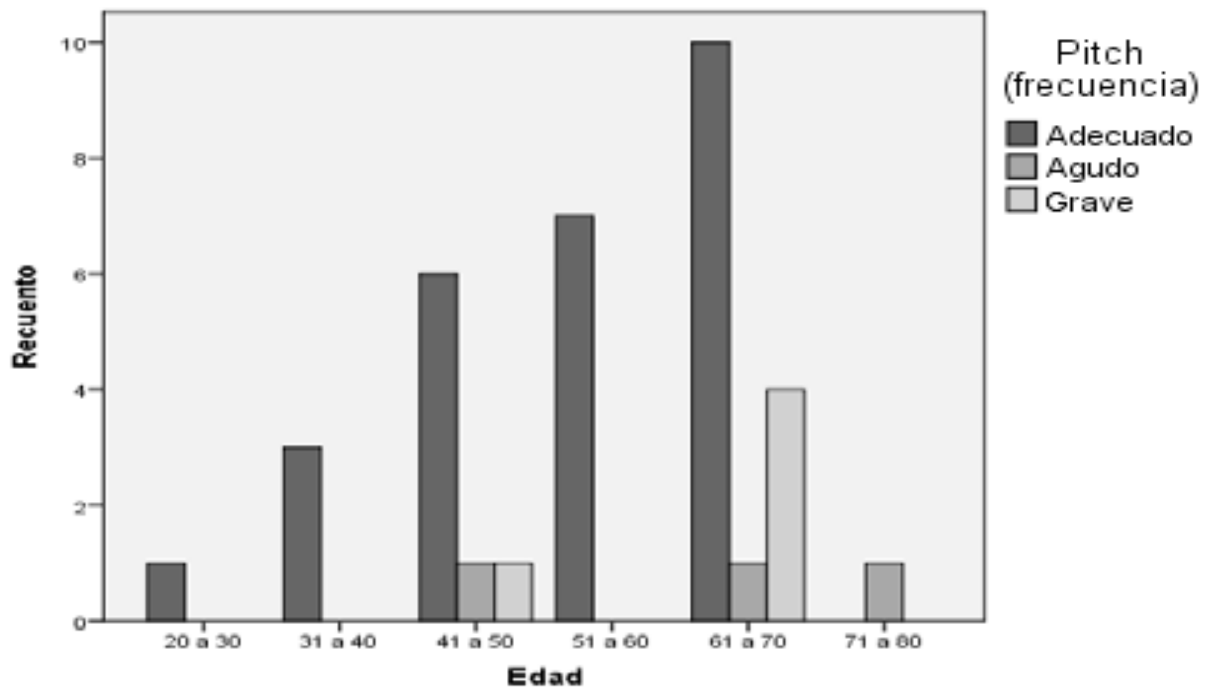

Figura 5. Relación de la edad con el nivel de pitch

La mayor parte de las docentes tuvo un nivel de pitch adecuado $(77.1 \%)$ de los cuales la mayoría tuvieron entre 61 y 70 años (37.0\%). Asimismo, las docentes que tuvieron niveles de pitch (frecuencia) agudo, según Mysak y Hamley (1958) hay aumento en la variabilidad del pitch. También se encontró en este grupo etéreo docentes cuyo pitch es grave. Los investigadores refieren la reducción de las frecuencias (pitch) fundamental de la voz para las mujeres (Saxman y Burk 1967; Kelley 1977; Alarcos, Behlau y Tosi 1963; Morrison y Rammage, 1994; Carbolle, Tolasa y Juan 1996).

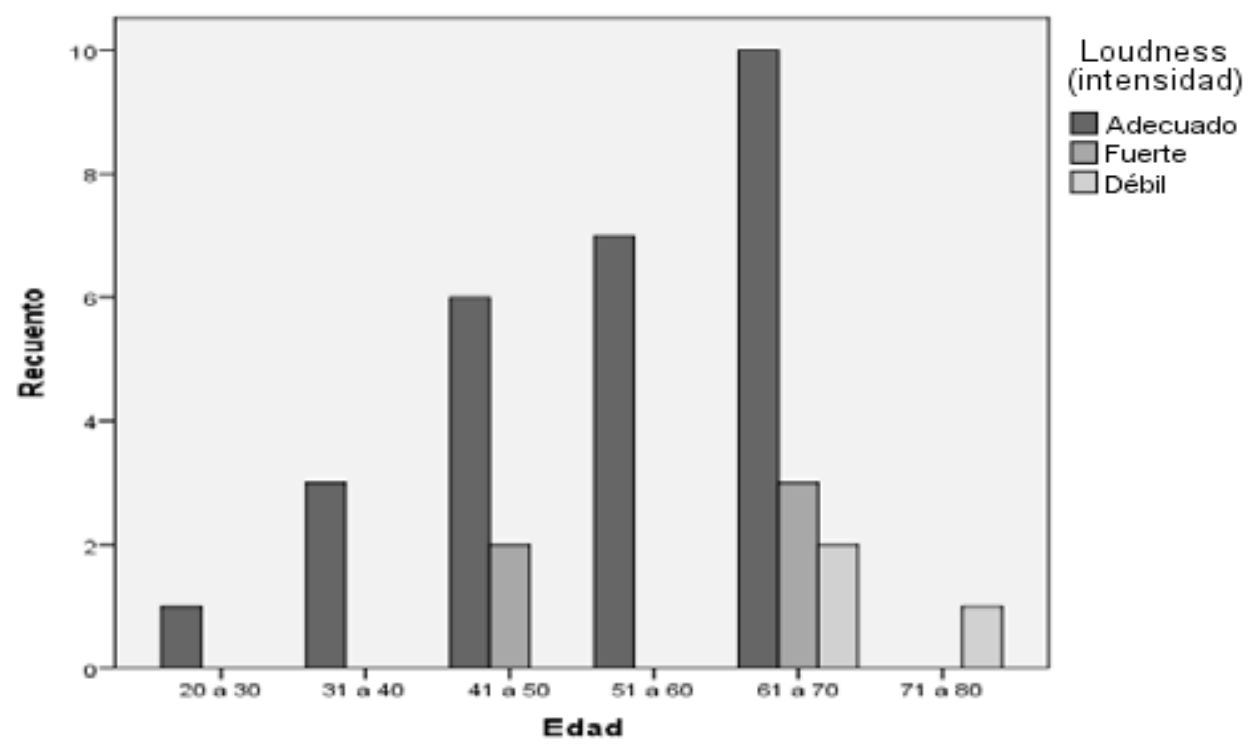

Figura 6. Relación de la edad con el nivel de loudness

El $77.1 \%$ de las profesoras tuvieron un nivel de laudness (instensidad) adecuada, de los cuales la mayoría tuvieron 61 a 70 años (37.0\%). Asimismo, los docentes que tuvieron niveles de Laudness fuerte y débil, estuvieron distribuidos en diferentes grupos etarios, siendo los más frecuentes en docentes entre 61 a 70 años. Leslie Piccolotto Ferreira, Marta de Andrade e Silva (2002) manifestaron que el pitch es considerado fuerte y débil también es conocido como potencia de voz, que se encontraron entre las edades anteriormente mencionadas. 


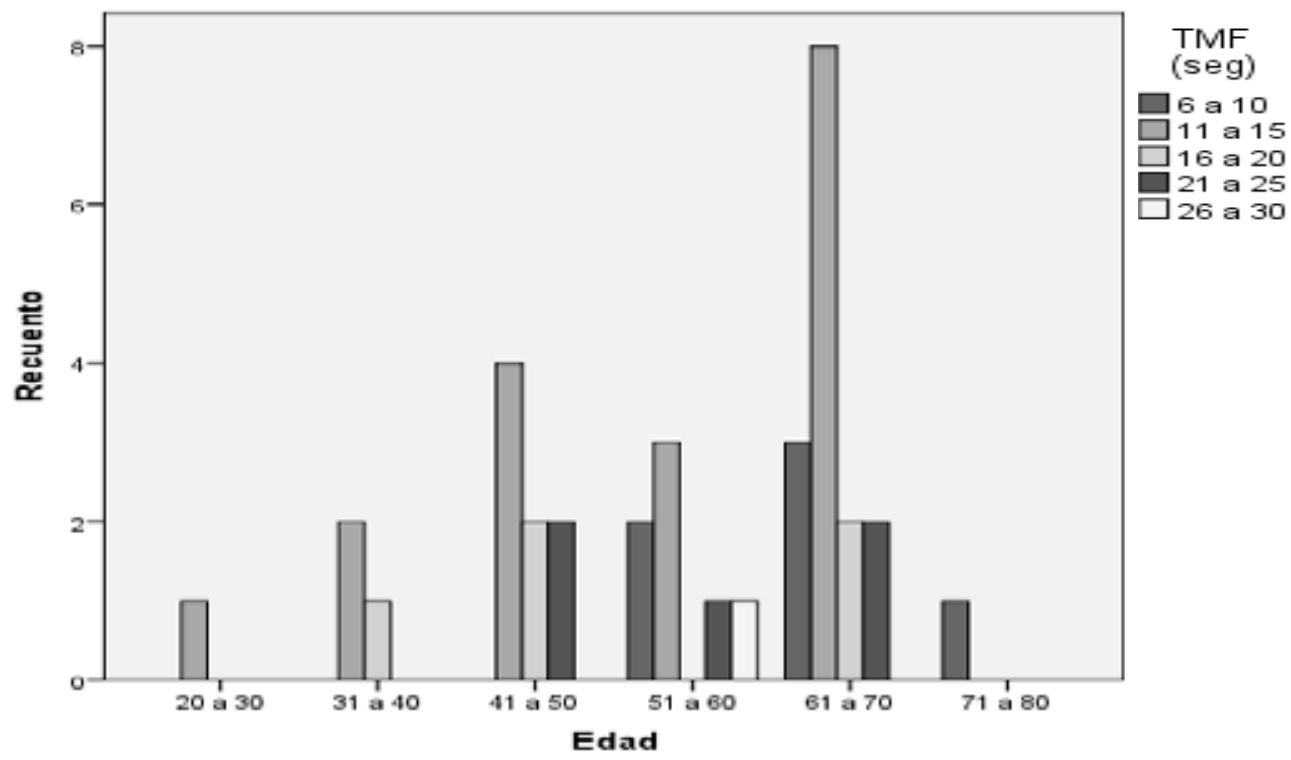

Figura 7. Relación de la edad con el tiempo máximo de fonación (TMF)

El tiempo máximo de fonación más frecuente en los sujetos fue de 11 a 15 segundos $(51.4 \%)$ de los cuales, el mayor porcentaje es presentado entre 61 y 70 años, con $44.4 \%$ del total de sujetos con el tiempo máximo de fonación. Mara Behlau y Paolo
Pontes (1995) consideraron como el tiempo máximo de fonación de 15 a 25 segundos para hablantes femeninas. En este cuadro los sujetos evaluados presentan un nivel inferior a las medidas establecidas entre las profesoras.

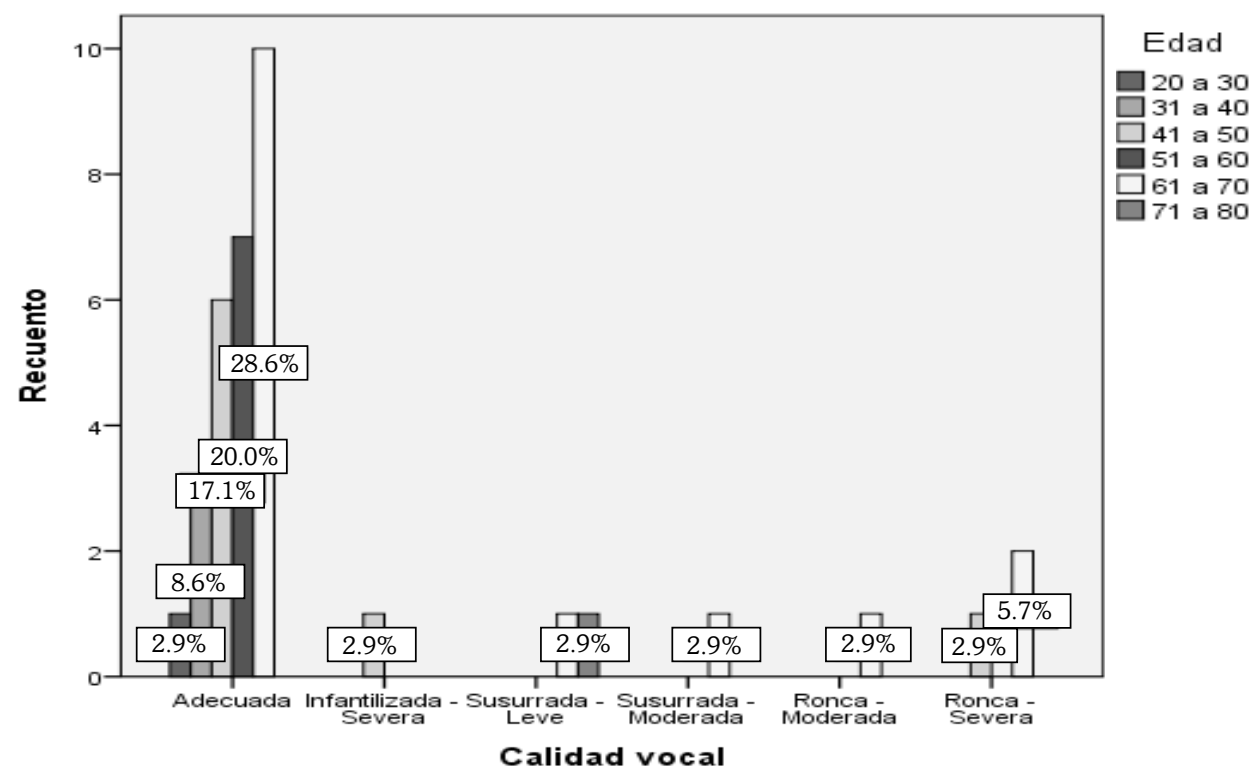

Figura 8. Relación de la edad con la calidad vocal

La mayor parte de las profesoras tuvieron una calidad vocal adecuada $(77.1 \%)$, porcentaje donde la mayoría tuvieron entre 61 y 70 años $(37.0 \%)$.

Asimismo, las profesoras que tuvieron diferentes alteraciones de calidad vocal estuvieron distribuidos en diferentes grupos etarios, siendo las más frecuentes las profesoras entre 61 a 70 años.

Según Mara Behlau (2001 todos los estudios científicos fracasan en la descripción de un marcador que fuese único y exclusivo para la voz del adulto mayor. 


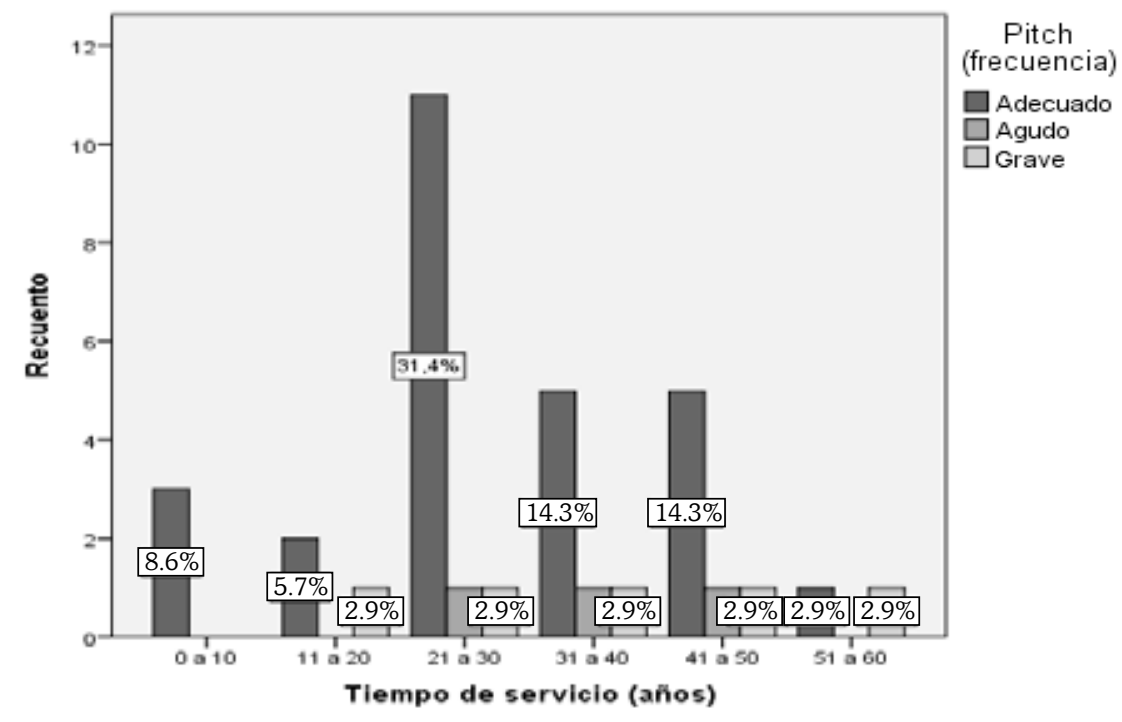

Figura 9. Relación del tiempo de servicio de los adolescentes con el nivel de pitch

Las profesoras presentan una frecuencia de voz adecuada en un $77.1 \%$; de la cual el $40.7 \%$ corresponde a docentes con 21 a 30 años de servicio seguido de las profesoras con 31 a 50 años de servicio (37.0\%. El 8.36\% de las profesoras presentaron una secuencia de voz aguda y corresponde a quienes tienen 21 a 50 años de servicio profesional. El $14.3 \%$ de las profesoras tuvieron una frecuencia grave $y$ entre 11 a 60 años de servicio. Para los autores Mara Behlau y Paulo Pontes la sensación psicoacúsica relacionada a la frecuencia o sea como juzgamos un sonido en la que respetamos su altura, considerando el más grave o el más agudo, depende básicamente de la frecuencia fundamental desde sonido, embora hoja también la influencia de la intensidad de la resonancia.

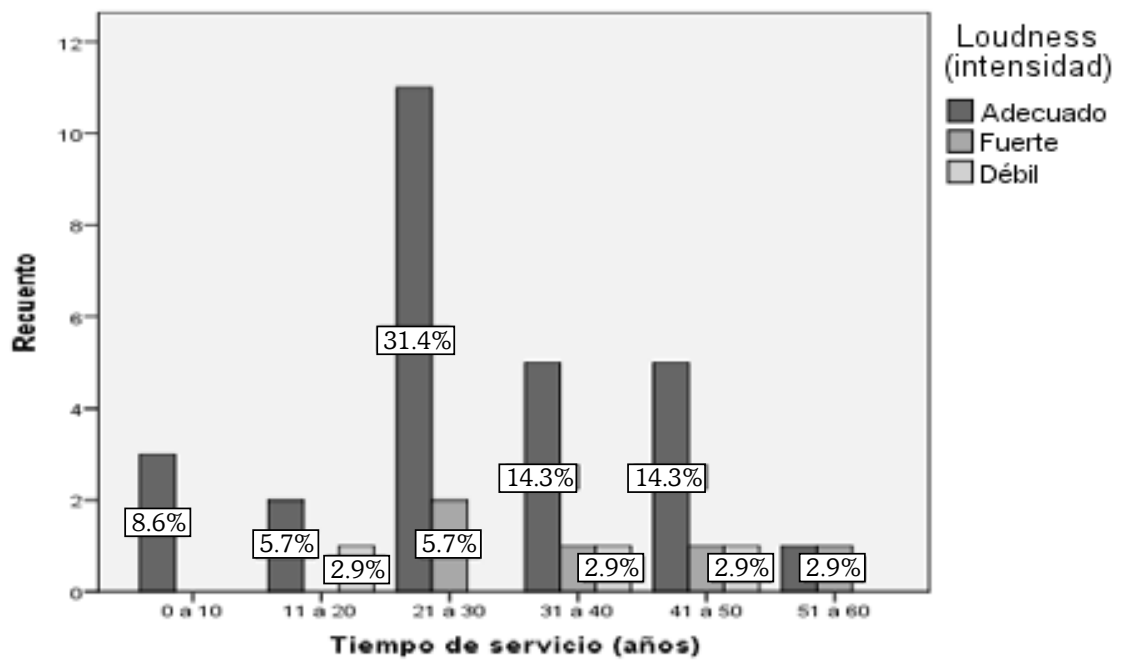

Figura 10. Relación del tiempo de servicios de las docentes con el nivel de loudness

Las profesoras tuvieron una laudness de voz adecuada con $77.1 \%$ de frecuencia, de los cuales el $40.7 \%$ correspondieron a docentes con 21 a 30 años de servicio, seguido de docentes con 31 a 50 años de servicio (37.0\%). El 14.3\% de los docentes presentaron una intensidad de voz fuerte, y estuvieron distribuidas en docentes con 21 a 60 años de servicio. El $8.6 \%$ de las profesoras tuvieron una intensidad de voz leve, y estuvieron distribuidos en docentes con 11 a 20 y 31 a 50 años de servicio Carbonell, Tolasa y Juan (1996) dicen que en la edad adulta el perfil de la extensión vocal presenta valores medios 
En otros casos se reduce la intensidad vocal en número de las notas de la tesitura vocal, por la presión intraoral máxima (Placek, Sanders, Maloney y Jackson, 1966).

\section{CONCLUSIONES}

En la presente investigación, en base a la evaluación de las docentes de la UNIFÉ se obtuvo los siguientes resultados:

1. Presentan el pitch (frecuencia), loudness (intensidad) y nivel de calidad vocal adecuado (ver figuras 1,2 y 4 ).

2. Con relación al tiempo máximo de fonación, solo $28.6 \%$ se ubica dentro del rango normal para mujeres, 15 a 25 segundos (ver figura 3).

3. En la interpretación de edades en relación al pitch se aprecia que el mayor porcentaje de docentes con el pitch adecuado, se ubica en el rango de 61 a 70 años (ver figura 5).

4. En este mismo rango se ubica el mayor porcentaje de docentes con un nivel de loudness y calidad de voz adecuado, $37.0 \%$ (ver figuras 6 y 8 ).

5. Con relación al tiempo de servicio se puede afirmar que el mayor porcentaje de docentes con pitch adecuado se ubica en el grupo de 21 a 30 años de servicio (40.0\%). También en este grupo, en la misma proporción, se ubica el mayor porcentaje de docentes con loudness adecuado (ver figuras 9 y 10 ).

6. Con relación al tiempo de servicio de las docentes y la calidad vocal adecuada, el grupo que presenta un mayor porcentaje $(100 \%)$ es que tiene 0 a 10 años de tiempo de servicio (figura 10). 


\section{REFERENCIAS}

Behlau, Mara; Pontes, Paulo \& Ponti (1995) Evaluación y Tratamiento de las Disfonías. (Edición 1er.) Sau Paulo: Lavise Ltda. Brasil.

Behlau, Mara (2001) Voz O Libro do Especialista, Volumen 1, Río de Janeiro, Brasil.

Behalu, Mara; María Luisa Suzigan Dragone; Lucía Nagano (2004) A Voz que ensina O Professor e a Comunicacao Oral em sala de aula. Sau Pauto, Editora Revinter Ltda.

Bojorquez R., Mariela; Rodriguez V., Sonia (2003) Tesis: Alteraciones vocales de los Profesores del CEENE Fernando Wiese Eslava y del Colegio Antares, CEPAL. Lima - Perú.

Casado \& Adrian, J. (2012) Evaluación Clínica de la Voz. España.

Granda T. María y Castañeda, Norma (1989) Tesis: Disfonías y tratamiento.

Grune \& Mathienson (1989) La voz y sus desórdenes. Londres, Inglaterra: Whurr

Farías, Patricia G. (2007) Ejercicios para restaurar la función vocal. Observaciones Clínicas Buenos Aires, Argentina.

Farías, Patricia (2002) "Las Disfonías Ocupacionales". 1ª Edición. Argentina: Ediciones Barcelona.

Piccolotto Leslie y Marta A. Andrade (2002) "Salud Vocal. Práctica cronológica" Editorial Roca Ltda. Brasil.

Vásquez Emilio, CEPAL "Selección de Lecturas” Editorial CEPAL.

Irma Perazos Elementos de Foniatría. Editorial Floria, Córdova, Buenos Aires.

Francois Le Huche - Halli Andre "La Voz. Patología Vocal: Semiología y disfonías funcionales" Tomo2. Editorial Masson.

Fecha de recepción: 28-09-16

Fecha de aceptación: 06-12-16 
